discussed by clinicians and implemented using PDSA cycles with iterative changes tested and analysed. $\mathrm{PH}$ form completion was re-audited monthly for a 6-month period.

Result. Baseline data showed $61.54 \%$ of patients had physical health forms completed $(\mathrm{n}=26$; $61.54 \%$ with $\mathrm{HbA1c}, 76.92 \%$ with cholesterol completed). Iterative changes and improvements included; (i) paper list to track PH form completion, (ii) table on Microsoft Word, (iii) Excel spreadsheet, and (iv) a conditionally formatted Excel spreadsheet. The conditionally formatted Excel spreadsheet was colour-coded to show completed elements as green and incomplete elements as red.

Paper lists increased PH completion to $84.85 \%(\mathrm{n}=33)$. Word table increased $\mathrm{PH}$ completion to $96.43 \%(\mathrm{n}=28)$. Excel spreadsheet had PH completion of $96.67 \%(\mathrm{n}=30)$. Colour coded excel spreadsheet increased PH completion to $100 \%(\mathrm{n}=28)$. This was used as standard practice with sustained $100 \%$ completion in November $(n=34)$ and December $(n=39)$. The improvement was sustained to January 2021, although there was a decrease to $97.7 \%(\mathrm{n}=30)$.

Conclusion. It was hypothesised an intervention to track completion of $\mathrm{PH}$ forms would improve completion rate. The use of a colour-coded conditionally formatted Excel spreadsheet improved $\mathrm{PH}$ form completion to $100 \%$ within an 8 -week period and a sustained increase of $>95 \% 6$ months after the study began. This study recommends the use of such an electronic record keeping system to assist with $\mathrm{PH}$ form completion.

\section{Increasing participation in the 2019 UK general election amongst patients on a high intensity rehabilitation ward}

\author{
Aaron Wood* and Amrith Shetty \\ Bowmere Hospital, Peasley Cross Hospital \\ ${ }^{\star}$ Corresponding author.
}

\section{doi: 10.1192/bjo.2021.608}

Aims. To increase participation in the 2019 UK general election amongst inpatients on a high intensity rehabilitation ward, by supporting patients to both register to vote (RTV) and vote.

Background. In 2000, the franchise was extended to those under section 2 or 3 as well as informal inpatients. Unfortunately, voting rates remain low: studies of the 2010 general election show voting rates amongst psychiatric inpatients to be $14 \%$, compared to $65 \%$ for the general population. Engaging patients in the democratic process is not only just, it has been shown to be an effective avenue for rehabilitation through increasing social capital. The 2019 UK general election represents a singular opportunity for biopsychosocial rehabilitation.

Method. In the three weeks up until 26/11/19 - the deadline to RTV - visual displays and verbal information were used to notify patients of:

\section{The election}

Their eligibility

The need to RTV before casting a ballot

The registration deadline

Voting methods (in person, by post, by proxy)

We gathered patients' intention to RTV and offered impartial, personalised support to register online or by paper, and to apply for a postal or proxy ballot if wished. Patients with no fixed abode were supported to use the ward as their declared place of residence.
Result. Of the 17 patients on the ward there were:

Four informal patients

11 patients under section 3

One patient each under a section 37 and a section 37/41, both ineligible to vote

Of the 15 eligible patients, one (6.7\%) had already registered, six patients (40\%) wanted to register and eight (53.3\%) stated they did not want to register. Those wanting to register were supported according to individual patient preference. Of the registered seven, five $(33.3 \%)$ reported voting, one $(6.7 \%)$ reported not having voted and one (6.7\%) declined to say. Two (13.3\%) voted in person and five $(33.3 \%)$ voted by postal ballot.

Conclusion. Our intervention corresponded with an increase in number of patients registering - from one patient $(6.7 \%)$ to seven $(46.7 \%)$, with 5-6 (33.3-40\%) casting their ballot. While the causal relationship should not be overstated, the uptake of assistance supports the intervention's efficacy.

Good rehabilitation increases a person's social capital, empowering them to actively participate in societal life. Registering to vote is a tacit assertion of this principle. Our study shows that brief interventions that are easily incorporated into everyday care are a simple, effective and ultimately necessary tool in holistic mental health rehabilitation.

Rates of cervical screening amongst females admitted to the psychiatric inpatient hospital in Jersey, Channel Islands

Jade Wright

St Saviours Hospital, Jersey

doi: 10.1192/bjo.2021.609

Aims. Patients with enduring mental health conditions are known to have higher morbidity and mortality rates than the general population. It has been identified that this is due to lifestyle risk factors, medication side effects and barriers to receiving physical health care. National screening programmes; including cervical screening, save lives, however depends upon patient engagement. We hypothesised that due to the factors stated above, psychiatric inpatients are more at risk of cervical cancer and less likely to engage in cervical screening. This study aimed to assess the cervical screening history of patients discharged from the psychiatric inpatient hospital in Jersey, Channel Islands.

Method. Using computerised laboratory records, the cervical smear history of female patients discharged from the paychiatric inpatient hospital was analysed. Inclusion criteria were: being aged between 25-64 years and having a cervix in situ. Exclusion criteria were total hysterectomy. Cervical smear history was compared to the national guidelines of having routine smears every 3 years for women aged 25-49 and every 5 years for women aged between 50-64 years.

Result. In the period 1 December 2019-1 December 2020 there were 45 females discharged from the psychiatric inpatient hospital that fit the inclusion criteria. 26 (58\%) were up to date with their cervical smears in accordance with national guidelines. 12 (27\%) had previously had a smear but were not up to date. 19 smears were done at the GP, 13 at the sexual health clinic and 6 at gynaecology clinic. 7 (16\%) had never had a cervical smear. Of these 7 patients it was identified that one patient was in a same sex relationship and one was a victim of sexual assault.

Conclusion. $58 \%$ of women discharged from the psychiatric inpatient hospital were up to date with their smears. This is down from the $72.2 \%$ coverage rate of the general population. Although this was a small study, it highlights that engagement 